\title{
Parameterization to NDDO-based polarizable force field
}

\author{
Heike Thomas ${ }^{1}$, Matthias Hennemann ${ }^{1}$, Stefan Guessregen², Timothy Clark ${ }^{1,3^{*}}$ \\ From 9th German Conference on Chemoinformatics \\ Fulda, Germany. 10-12 November 2013
}

In Computer-Aided-Drug-Design (CADD), the electrostatic interactions contribute strongly to the interaction between the drug-molecule and the target. Further, the Coulomb term is crucial for calculating the electrostatic contribution to the solvation energy. In spite of this, conventional Force Fields use the obsolete physical concept of point-monopoles (net atomic charges) and thus, are not able to represent the molecular electrostatic potential (MEP) accurately or are even wrong for atoms that have positively and negatively charged areas on their surface [1]. A far better way to describe the MEP is the is multipole-based semiempirical MO-theory [2,3]. For the parameterization of the polarizable hpCADD Force Field, the two methods are combined in order to obtain both the MEP and structures and energies. Additionally, the differentiation of atom-types leads to more detailed information about the MEP.

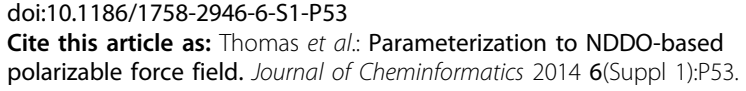

Cite this article as: Thomas et al:: Parameterization to NDDO-based polarizable force field. Journal of Cheminformatics 2014 6(Suppl 1):P53.

\begin{abstract}
Authors' details
${ }^{1}$ Computer-Chemie-Centrum, Friedrich-Alexander-Universität ErlangenNürnberg, Nägelsbachstraße 25, 91052 Erlangen, Germany. ${ }^{2}$ Sanofi Deutschland GmbH, R\&D, LGCR, Structure, Design and Informatics, Building G878, 65926 Frankfurt am Main, Germany. ${ }^{3}$ Centre for Molecular Design, University of Portsmouth, King Henry Building, Portsmouth PO1 2DY, UK.
\end{abstract}

Published: 11 March 2014

\section{References}

1. Politzer $P$, Murray JS: $\sigma$-Hole bonding between like; a fallacy of atomic charges. J Mol Model 2008, 14:659-665.

2. Devereux M, Plattner N, Meuwly M: Application for multipolar charge models and molecular-dynamic simulations to study Stark shifts in inhomogeneous electric fields. J Phys Chem A 2009, 113:13199-13209.

3. Dewar MJS, Thiel W: Ground states of molecules. 38. The MNDO method. Approximations and parameters. J Am Chem Soc 1977, 99:4899-4907.

\footnotetext{
* Correspondence: Tim.Clark@fau.de

${ }^{1}$ Computer-Chemie-Centrum, Friedrich-Alexander-Universität Erlangen-

Nürnberg, Nägelsbachstraße 25, 91052 Erlangen, Germany

Full list of author information is available at the end of the article
}

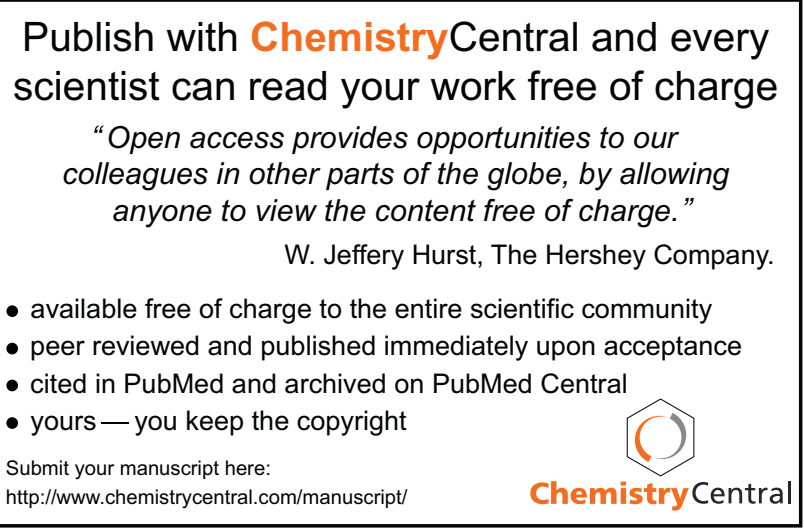

